DOI 10.18699/PlantGen2019-119

\title{
Duplication of the dominant Vrn-A1b.2 allele \\ in Triticum dicoccum lineage
}

\author{
Muterko A. \\ Institute of Cytology and Genetics, SB RAS, Novosibirsk, Russia \\ e-mail:muterko@gmail.com
}

The Triticum dicoccum lineage includes domesticated hulled tetraploid wheat of T. dicoccum and hexaploid wheat species of T. spelta, T. macha and T. vavilovii, which were derived from hybridisation events between the T. dicoccum and free-threshing hexaploid wheat of T. aestivum. The dominant VERNALIZATION-A1 alleles determine spring growth habit (without vernalization requirement) and early flowering of wheat. The Vrn-Alb.2 allele is frequent in tetraploid wheat of T. dicoccoides and T. dicoccum as well as in hexaploid wheat of T. spelta. In T. spelta this allele as well as Vrn-BIC are major determinants of the spring phenotype, while T. macha and T. vavilivii, as a rule, are characterized the strong vernalization requirement (winter type) and carry the recessive $V R N 1$ genes. The VRNI-ratio test, based on end-point qPCR was optimized to estimate the copy number variation (CNV) of VRN1 in wheat. Applied to the analysed accessions this test showed a two-fold increase in signal for the VRN-Al fragments in two accessions of $T$. dicoccum from Israel and Palestine carrying vrn-Alb.3 and numerous accessions of T. spelta from Europe carrying Vrn-Alb.2 and vrn-Alb.3. This difference in amplification of the $V R N-A l$ fragments was preserved at different level of the genomic DNA fragmentation, excluding the genomic environment effect, and was confirmed during TaqMan real-time PCR assay with the different endo- and exogenous controls. Duplication of Vrn-Alb.2 in accessions of T. spelta was strongly associated with the Vrn-A1b.2/Vrn-BIc genotype and awned spikes, indicating the bottleneck and founder effects. It is known that gene dosage of the dominant $V R N 1$ alleles positively correlates with early flowering of polyploid wheat. On the other hand, multiplication of the recessive $v r n-A l$ is associated with the later flowering. In any case the effect of the $V r n-A 1 b .2$ duplication on phenotype provides additional advantages in manipulation of the flowering time of wheat.

Acknowledgements: This study was supported by the Russian Science Foundation. This research was funded by the Russian Scientific Foundation grant No. 18-74-00080. 